ARTICLE

DOI: $10.1038 / \mathrm{s} 41467-017-01144-9$

\title{
The giant protein titin regulates the length of the striated muscle thick filament
}

Paola Tonino ${ }^{1,2}$, Balazs Kiss ${ }^{1,2}$, Josh Strom¹,2, Mei Methawasin ${ }^{1,2}$, John E. Smith III (10 1,2, Justin Kolb ${ }^{1,2}$, Siegfried Labeit ${ }^{3,4} \&$ Henk Granzier ${ }^{1,2}$

The contractile machinery of heart and skeletal muscles has as an essential component the thick filament, comprised of the molecular motor myosin. The thick filament is of a precisely controlled length, defining thereby the force level that muscles generate and how this force varies with muscle length. It has been speculated that the mechanism by which thick filament length is controlled involves the giant protein titin, but no conclusive support for this hypothesis exists. Here we show that in a mouse model in which we deleted two of titin's Czone super-repeats, thick filament length is reduced in cardiac and skeletal muscles. In addition, functional studies reveal reduced force generation and a dilated cardiomyopathy (DCM) phenotype. Thus, regulation of thick filament length depends on titin and is critical for maintaining muscle health.

\footnotetext{
${ }^{1}$ Department of Cellular and Molecular Medicine, University of Arizona, Tucson, Arizona 85721, USA. ${ }^{2}$ Sarver Molecular Cardiovascular Research Program, University of Arizona, Tucson, Arizona 85721, USA. ${ }^{3}$ Department of Integrative Pathophysiology, Medical Faculty Mannheim, Mannheim 68167, Germany. ${ }^{4}$ DZHK, Mannheim-Heidelberg 68167, Germany. Paola Tonino and Balazs Kiss contributed equally to this work. Correspondence and requests for materials should be addressed to H.G. (email: granzier@email.arizona.edu)
} 
$\mathrm{T}$ he contractile machinery that powers striated muscle (heart and skeletal muscles) has as its most crucial component the thick filament, comprised of the molecular motor myosin ${ }^{1,2}$. The thick filament is of a precisely controlled length ${ }^{3}$, defining thereby the force level that muscles generate and how this force varies with muscle length ${ }^{4}$. The mechanisms by which the thick filament length is so exquisitely controlled are unclear, and it has been speculated that the giant protein titin could be involved and function as a molecular 'ruler ${ }^{3-8}$.

Titin, the largest protein known, spans the half-sarcomere (contractile unit of muscle), from Z-disk to M-band ${ }^{9}$, is modular in structure, and contains $~ 300$ immunoglobulin (Ig)- and fibronectin (Fn)-like domains. The I-band segment of titin contains only Ig domains and several unique sequences ${ }^{10}$, all of which contribute to titin's elasticity that allows it to function as a complex molecular spring that contributes greatly to the diastolic stiffness of the heart ${ }^{11}$. This spring can be tuned with as prominent tuning mechanism post-transcriptional regulation that results in isoforms with distinct spring region composition ${ }^{12,13}$. The adult heart coexpresses the small and relatively stiff N2B titin isoform and the longer and more complaint N2BA titin isoform ${ }^{14}$.

Compared to titin's I-band region, its A-band segment is not well understood, yet recent landmark sequencing studies in large patient cohorts show that these zones are crucial as countless mutations linked to cardiac and skeletal muscle diseases are found here ${ }^{12,15-18}$. Titin's A-band segment is orders of magnitude less extensible than the I-band region of the molecule $^{19}$ and it is unlikely therefore that the A-band segment of titin functions as a molecular spring. Titin's A-band segment largely consists of Ig and Fn domains that form a 7-domain fixed pattern in the D-zone and an 11-domain fixed pattern in the C-zone (see Fig. 1a with domain organization based on ref. ${ }^{10}$ ). The C-zone is most prominent and contains 11 super-repeats of the Ig-Fn-Fn-Ig-Fn-Fn-Fn-Ig-Fn-Fn-Fn pattern. Each super-repeat spans $\sim 43 \mathrm{~nm}$ in length ${ }^{20}$, binds to myosin ${ }^{21}$ and myosin-binding protein-C (MyBP-C $)^{22}$, and is referred to as a C-zone repeat ${ }^{10}$. Titin molecules run along the thick filament and each of its super-repeats spans $\sim 43 \mathrm{~nm}$ in length, a distance that coincides with the $\sim 43 \mathrm{~nm}$ myosin helical repeat ${ }^{20}$. Hence, a popular but untested theory is that in vertebrate animals titin functions as a thick filament template that is responsible for determining thick filament length. A recent study in which a large part of titin near the edge of the A-band was deleted was negative in that the thick filament length was unaltered $^{19,23,24}$.

Here we report studies on a mouse model, $T t n^{\Delta C 1-2}$, in which two of titin's super-repeats (C1 and C2) were deleted. Structural studies in both cardiac and skeletal muscles of $T t n^{\Delta C 1-2}$ mice reveal a reduced thick filament length, in line with the concept of a $2 \times 43 \mathrm{~nm}$ shortened titin ruler. Skeletal muscles of $T t n^{\Delta C 1-2}$ mice generate less force and have a steeper descending limb of their force-sarcomere length relation, supporting the structural finding of shorter thick filaments. The heart generates less pressure and, unexpectedly, has a dilated cardiomyopathy (DCM) phenotype, a heart disorder characterized by ventricular dilation and depressed contractility ${ }^{25}$ and a common cause of heart failure in humans with a prevalence of up to $1: 250^{26}$. Importantly, there are many truncation mutations in the A-band segment of titin (including 12 within the $\mathrm{C} 1$ and $\mathrm{C} 2$ repeats) associated with $\mathrm{DCM}^{15,27,28}$ and these truncation mutations may impact titin's role in thick filament length regulation, causing a force reduction and leading to DCM. Thus, our work shows for the first time that thick filament length regulation is titin based and is essential for maintaining muscle health.

\section{Results}

The $T t n^{\Delta C 1-2}$ mouse model. To test the role of titin in thick filament length regulation, homologous recombination was used to delete from the mouse titin gene exons 305-325 (details in Supplementary Fig. 1A). This deletion keeps the reading frame intact but internally deletes from titin 2177 amino acids $(239.5 \mathrm{kDa})$ that code for titin's C-zone super-repeats 1 and 2 . The homozygous $T t n^{\Delta C 1-2}$ mice are viable and are obtained from heterozygous parents at a Mendelian ratio (Supplementary Fig. 1B). $\mathrm{Ttn}^{\Delta C 1-2}$ mice have normal growth curves and muscle weights (Supplementary Fig. 1C, D). An RNA sequencing (RNAseq) analysis revealed that titin exon usage in the adult myocardium is unaltered, except for the absence of the deleted exons 305-325, indicating that no adaptations in splicing occur (Fig. 1b). Protein analysis on both left ventricular (LV) myocardium and extensor digitorum longus (EDL) skeletal muscle revealed that homozygous mice express titin of increased mobility (Fig. 1c), but at normal levels (Supplementary Fig. 2A, B), with also normal myosin expression (Supplementary Fig. 2B-D). An exception is the small but significant upregulation of N2BA titin at the expense of $\mathrm{N} 2 \mathrm{~B}$ titin in the left ventricle of $T t n^{\Delta C 1-2}$ mice. Western blots using antibodies to titin's $\mathrm{N}$ terminus (Z1Z2) and $\mathrm{C}$ terminus (M8-9) show that these regions are normally expressed in mutant titin (Fig. 1c). Additionally, superresolution optical microscopy (structured illumination microscopy (SIM)) reveals that titin's $\mathrm{N}$ and $\mathrm{C}$ termini are normally incorporated in the sarcomere of $T t n^{\Delta C 1-2}$ mice (Fig. 1d). Thus, except for the internal deletion of two super-repeats, the titin molecule in $T t n^{\Delta C 1-2}$ mice is intact. The $T t n^{\Delta C 1-2}$ model that we created is ideal for studying the role that titin plays in thick filament length regulation.

Thick filament length. Structural studies were focused on left ventricular cardiac muscle and EDL skeletal muscle of 8-week-old homozygous $T t n^{\Delta C 1-2}$ and littermate wild-type (WT) control mice. In initial studies, the thick filament length was determined from the width of the A-band as measured by electron microscopy (EM). Studies on cardiac muscles stretched by different degrees revealed that the A-band width was significantly reduced in $T t n^{\Delta C 1-2}$ mice (Supplementary Fig. 3A), with similar findings in EDL muscle (Supplementary Fig. 3B). Immunoelectron microscopy (IEM) was performed next, using the I103 titin antibody that is known in WT muscle to mark titin at the end of the thick filament ${ }^{19}$ near the junction between the A-band and the I-band (location of its binding site in titin is shown in Fig. 1a, see also ref. ${ }^{19}$ ). If titin does not regulate thick filament length, then deleting C-zones 1 and 2 is expected to move the I103 epitope inside the A-band by $\sim 86 \mathrm{~nm}$ (twice the length of the C-zone repeats). However, in $\mathrm{Ttn}^{\Delta \mathrm{Cl} 1-2}$ mice the $\mathrm{I103}$ epitope maintained its location near the edge of the A-band in both cardiac and skeletal muscle (compare left and middle panels of Supplementary Fig. 3C, D) and the graph of epitope-to-epitope distances versus sarcomere length was shifted to shorter distances in the $T t n^{\Delta C 1-2}$ mice, similar to the shift in A-band width (right panels of Supplementary Fig. 3C, D). Thus, deleting two C-zone repeats shortens both titin and the thick filament by similar amounts.

Preparing muscle tissue for transmission electron microscopy (TEM) is known to cause shrinkage during sample preparation ${ }^{3}$; to address whether the reduced thick filament length in $\mathrm{Ttn}^{\Delta \mathrm{Cl} 1-2}$ mice might be due to differential shrinkage, two distinct methods were used. The first was based on SIM, a method that unlike EM does not require dehydration and embedding and shrinkage is expected to be different from that in EM studies. For these studies, the I103 antibody was used as it marks the edge of the 
a

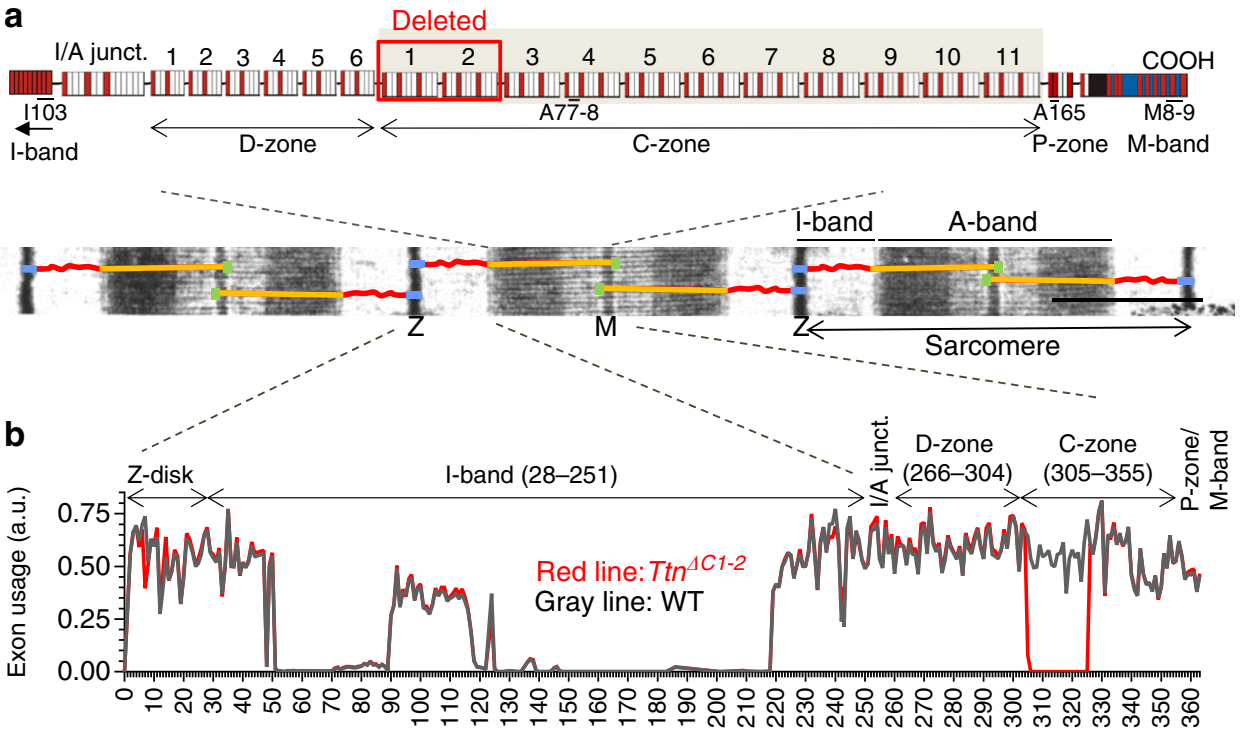

Titin exon

C

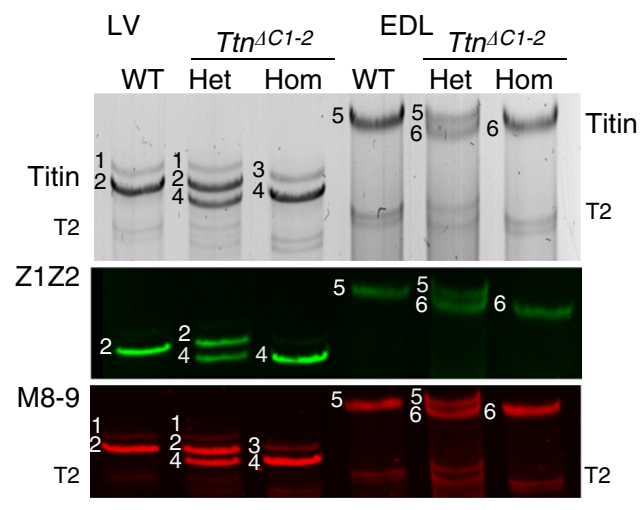

d
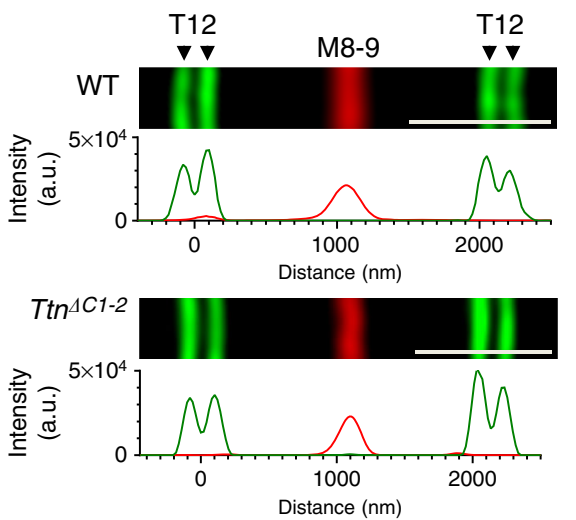

Fig. 1 Genetically engineered mouse model lacking two C-zone repeats in titin. a Titin spans from Z-disk (Z) to M-band (M) in the sarcomere. Top, domain structure of A-band segment of titin highlighting the C-zone and the two deleted C-repeats in the $\mathrm{Ttn}^{\Delta \mathrm{C} 1-2}$ mouse model (additionally, showing binding sites of the titin antibodies used in this study). b Titin exon usage in myocardial tissue from 8-week-old WT and $\operatorname{Ttn}^{\Delta C 1-2}$ male mice $(n=8$ each). c Top, agarose protein gel of titin in left ventricular (LV) myocardium and extensor digitorum longus (EDL) skeletal muscle. Bottom, western blots using Z1Z2 and M8-9 antibodies. 1-4 Cardiac titin (1 N2BA (WT), 2 N2B (WT), 3 N2BA (Ttn ${ }^{\Delta C 1-2}$ ), and 4 N2B (Ttn ${ }^{\Delta C 1-2}$ )); 5-6 EDL muscle titin (5 N2A (WT) and 6 N2A $\left.\left(T t n^{\Delta C 1-2}\right)\right)$. d Superresolution microscopy using T12 and M8-9 antibodies. Scale bars, $1 \mu \mathrm{m}$

A-band in both WT and Ttn ${ }^{\Delta C 1-2}$ mice (see above). Epitope-toepitope distances were found to be reduced in both muscle types of Ttn ${ }^{\Delta C 1-2}$ mice (Fig. 2a), by on average $164 \mathrm{~nm}$ in cardiac muscle and $163 \mathrm{~nm}$ in skeletal muscle, or $\sim 41 \mathrm{~nm}$ per deleted $\mathrm{C}$-zone repeat. Additionally, we performed IEM with a cardiac MyBP-C (cMyBP-C) antibody that labels multiple stripes in the A-band that in vivo are known to be $43 \mathrm{~nm}$ apart ${ }^{29}$. By measuring the stripe distance on electron micrographs we determined the degree of tissue shrinkage and used this to correct the measured A-band width values. Large shrinkage values were found in sarcomeres that are at short sarcomere length (Supplementary Fig. 4), consistent with short A-band findings of others ${ }^{30}$. Using cMyBP-C as an 'internal caliper' reveals that thick filament length was reduced in $\mathrm{Ttn}^{\Delta \mathrm{Cl}-2}$ myocardium (Fig. $2 \mathrm{~b}$ ), by $173 \mathrm{~nm}$ or $\sim 43 \mathrm{~nm}$ per deleted C-zone repeat (Table 1).

The available skeletal muscle-specific anti MyBP-C antibodies did not provide adequate labeling in EDL muscle, and therefore an alternative shrinkage correction method was developed. An antibody was raised against the first two Fn domains of C-zone 4 (A77-8, Fig. 1a); the real distance between the two epitopes across the M-band can be estimated at $892 \mathrm{~nm}$ (for details, see the legend of Supplementary Fig. 5C) and the deviation from the predicted value on electron micrographs was used to determine A-band shrinkage (for details, see Supplementary Fig. 5). Using A77-8 as the 'internal caliper' resulted in a thick filament length that was reduced in EDL muscle of $T t n^{\Delta C 1-2}$ mice (Fig. 2c, right) by $168 \mathrm{~nm}$ or $42 \mathrm{~nm}$ per deleted C-zone repeat (Table 1). Thus, a range of techniques clearly reveal that deleting two super-repeats from the titin gene results in shorter thick filaments, and the shrinkage-corrected values of $\sim 41-43 \mathrm{~nm}$ per half thick filament support the hypothesis that each titin super-repeat specifies $43 \mathrm{~nm}$ length increments of the thick filament.

Functional studies. The reduced thick filament length is expected to lower maximal calcium-induced force by $12 \%$ (taking the length reduction at $170 \mathrm{~nm}$ and the crossbridge bearing part of the thick filament at $1440 \mathrm{~nm}^{4}$ ). Furthermore, the descending limb of the force-sarcomere length relation (with force normalized to the maximal force at optimal sarcomere length) is 
a Cardiac

WT

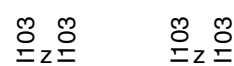

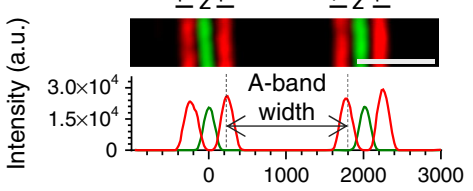

Distance $(\mathrm{nm})$

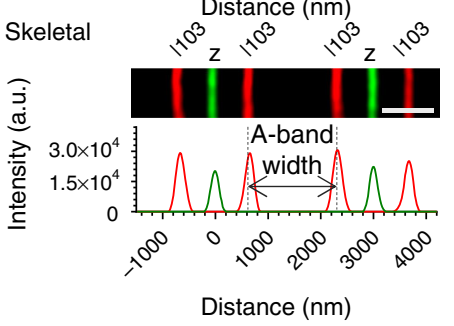

b Cardiac

WT
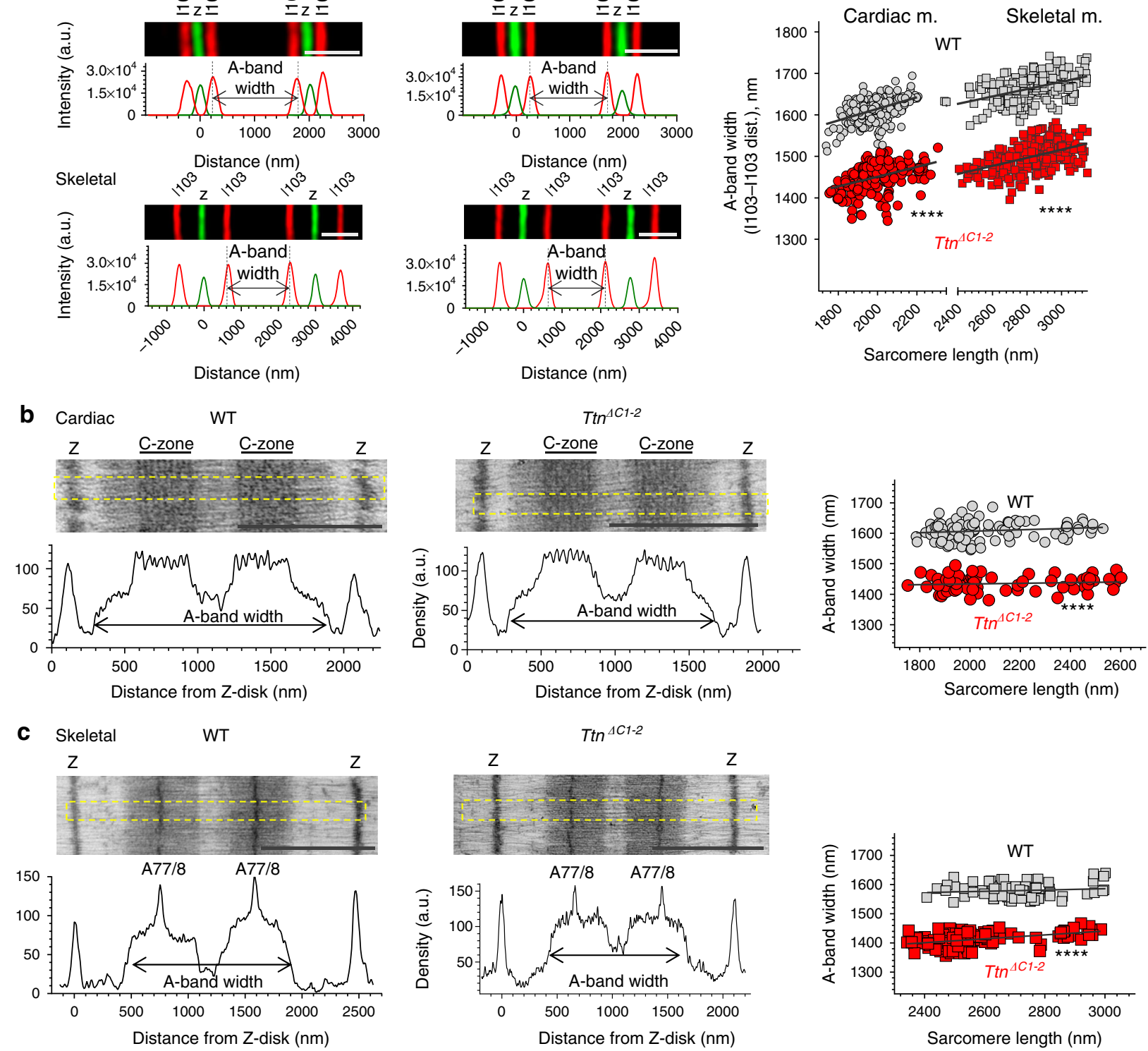

Distance (nm)

Sarcomere length $(\mathrm{nm})$

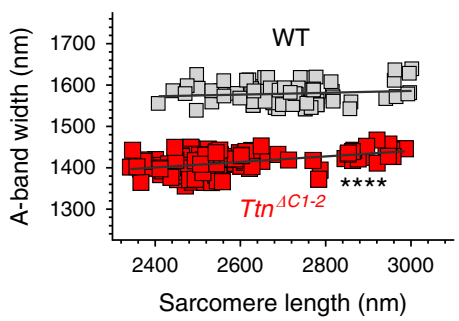

Fig. 2 Effects of deleting C-zone repeats on A-band width. a Left, example of SIM images obtained using cardiac and skeletal muscle sections labeled with 1103 titin antibody (A-band, red) and $\alpha$-actinin (Z-disk, green). Right, summarized results of the A-band width measurements. In both muscle types the offsets of the linear regression lines are significantly lower in $T t^{\Delta}{ }^{\Delta C 1-2}$ mice. $\mathbf{b}, \mathbf{c}$ Left, representative IEM images of sarcomeres labeled with cMyBP-C (b) or A77/8 (c) with density profiles shown at bottom. Obtained summarized results are shown to the right. c The distance between A77/8 epitopes was measured and used to determine A-band shrinkage in skeletal muscle. Right, Shrinkage-corrected A-band width results. All linear regression fits show positive slopes but none are significantly different from zero. Line offsets are significantly reduced in $T t^{\Delta C 1-2}$ mice. Statistical analysis is provided in

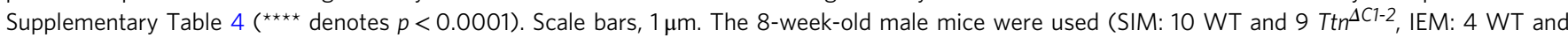
$4 \operatorname{Ttn}^{\Delta(1-2)}$

Table 1 Summary of the A-band width measurements based on IEM

\begin{tabular}{|c|c|c|c|c|c|c|}
\hline & \multicolumn{3}{|c|}{ Cardiac muscle } & \multicolumn{3}{|c|}{ Skeletal muscle } \\
\hline & WT & $T \operatorname{tn}^{\triangle C 1-2}$ & $p$-value & $\mathbf{W T}$ & $T \operatorname{tn}^{\Delta C 1-2}$ & p-value \\
\hline SL range $(\mu \mu)$ & $\sim 1.8-2.5$ & $\sim 1.8-2.6$ & & $\sim 2.4-3.0$ & $\sim 2.4-3.0$ & \\
\hline A-band width (nm) & $1608 \pm 30$ & $1435 \pm 25$ & $<0.0001$ & $1580 \pm 24$ & $1412 \pm 24$ & $<0.0001$ \\
\hline$\Delta$ A-band width $(\mathrm{nm})$ & & $173 \pm 4$ & & & $168 \pm 10$ & \\
\hline$\Delta$ per $C$-zone repeat $(\mathrm{nm})$ & & 43.4 & & & $42.0^{-}$ & \\
\hline
\end{tabular}



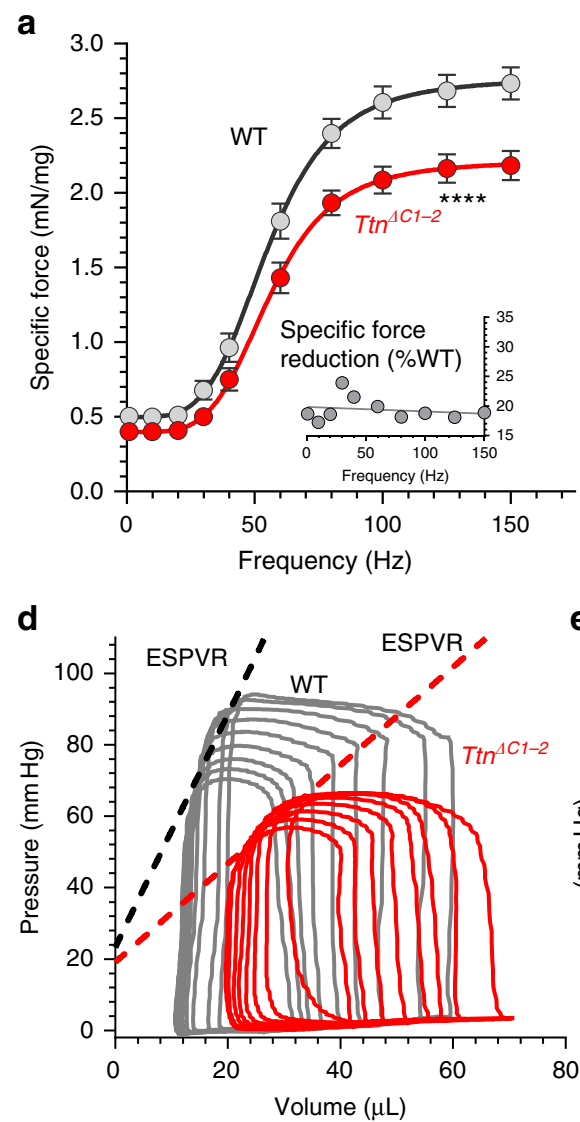

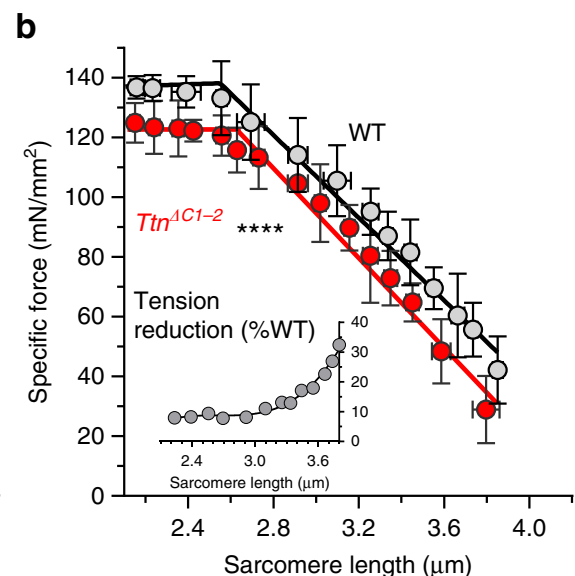

e

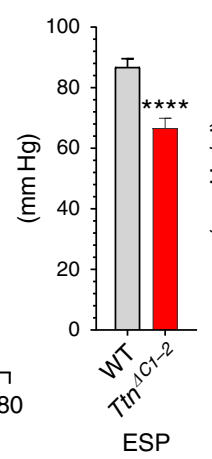

Pressure-volume analysis

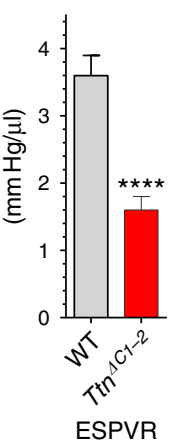

C

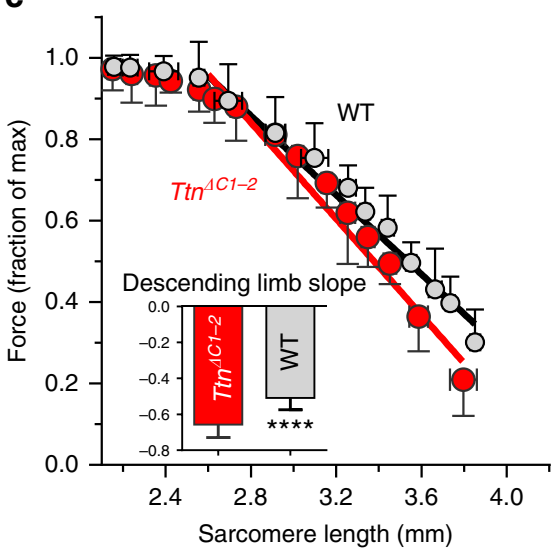

f

Echocardiography

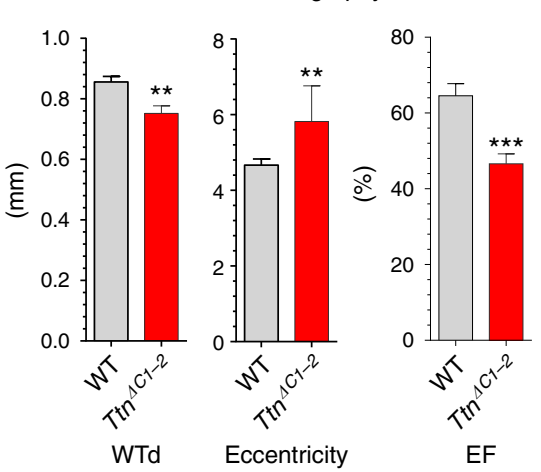

Fig. 3 Functional effects of deleting C-zone repeats of skeletal muscle and the heart. a In vivo force generation by the gastrocnemius muscle complex. The specific force frequency relation revealed significantly reduced force levels in $\operatorname{Ttn}^{\Delta C 1-2}$ mice (see inset). b, c Force-sarcomere length relations of skinned fiber bundles from EDL muscle activated by maximal calcium levels. b Specific force is significantly reduced in $T t^{\Delta C 1-2}$ mice (red symbols) with a reduction that, relative to WT levels, increases with sarcomere length (inset). c Force normalized to maximal force at optimal length reveals a descending limb with a slope that is significantly steeper in $T t n^{\Delta C 1-2}$ mice (inset). Mice at 60 days of age were used ( $n=6$ male and 2 female WT; $n=7$ male and 2 female $T t n^{\Delta C 1-2}$ ). d, e Pressure-volume (PV) analysis of the left ventricular chamber. Sample PV loops in Fig. 3d reveal reduced end-systolic pressures and a reduced slope of the end-systole PV relation (ESPVR) in $T \operatorname{tn}^{\Delta C 1-2}$ mice (red), with summarized results in Fig. 3e. Male mice at 62-74 days of age were used ( $n=7$ WT and $n=8 T t n^{\Delta C 1-2}$ ). $\mathbf{f}$ Echocardiography revealed wall thinning (diastolic wall thickness (WTd)), an increased eccentricity index, and a reduced ejection fraction (EF), all indicating that $T t^{\Delta C 1-2}$ mice have dilated cardiomyopathy (DCM). The 50-day-old male mice were studied $\left(n=8\right.$ WT and $n=8 T t n^{\Delta C 1-2}$ ). Mean values \pm s.e.m. are shown. Additional statistical analysis is provided in Supplementary Table 4 ( ${ }^{\star \star}$ denotes $p<0.01$; ${ }^{\star \star \star} p<0.001$, and $\left.{ }^{\star \star \star \star} p<0.0001\right)$

expected to be steeper due to the reduced length of the crossbridge bearing region of the thick filaments that reduces the distance for thin filaments to slide from $100 \%$ to $0 \%$ overlap. To test these predicted effects on force, we first carried out an in vivo functional study on skeletal muscle in which the gastrocnemius muscle was stimulated at a range of frequencies $(1$ to $150 \mathrm{~Hz}$ ). This revealed a significantly reduced force level in $T t n^{\Delta C 1-2}$ mice (Fig. 3a), on average $19.4 \pm 1.4 \%$ (Fig. 3a, inset). Studies were also conducted on demembranated fibers from the EDL muscle that were stretched to a predetermined sarcomere length and then activated with a maximal activating level of calcium $(0.1 \mathrm{mM}$ free $\mathrm{Ca}^{2+}$ ). This revealed that calcium-activated force at all sarcomere lengths on the plateau of the force-sarcomere length relation is reduced in $T t n^{\Delta C 1-2}$ fibers by on average $9.1 \pm 0.9 \%$ (Fig. $3 b$ ). The descending limb of the force-sarcomere length is down-shifted and when forces are expressed relative to WT levels, the force deficit progressively increases with sarcomere length (Fig. 3b, inset), consistent with the descending limb of the normalized force-sarcomere length relation that is steeper (Fig. 3c). These findings support the reduced thick filament length measured in skeletal muscles of $T t n^{\Delta C 1-2}$ mice.
To evaluate cardiac function, a pressure-volume analysis was conducted by inserting a small $1.2 \mathrm{~F}$ conductance catheter into the LV of the heart and recording pressure-volume (PV) loops at a range of end-diastolic volumes (see Methods for details). Sample loops are shown in Fig. 3d and summarized results in Fig. $3 e$ and Table 2. The end-systole pressure (ESP) is reduced in the $T t n^{\Delta C 1-2}$ mice as is the slope of end-systolic pressure-volume relation (ESPVR) and the preload recruitable stroke work (PRSW), both indexes of myocardial contractility ${ }^{31}$ (Fig. 3e, middle and right). PV studies also revealed that the LV operates at larger volumes in $T t n^{\Delta C 1-2}$ mice (Table 2). An echocardiography study showed in $T t n^{\Delta C 1-2}$ mice LV wall thinning, an increased eccentricity index (Fig. 3f, left and middle, Supplementary Table 1), and a reduced ejection fraction (Fig. 3f, right). These findings indicate that $T t n^{\Delta C 1-2}$ mice have DCM, a disorder characterized by LV chamber dilation and contractile dysfunction $^{25}, 32$.

To determine whether depressed contractility might be explained by changes in calcium handling in the $T t n^{\Delta C 1-2}$ mice, calcium transients were measured in isolated single cardiac myocytes that were twitch activated. No differences were found in the base level of calcium, the calcium amplitude, or in the kinetics 
of calcium release or uptake (Supplementary Fig. 6 and Supplementary Table 2), supporting that the DCM phenotype is primarily myofilament based.

The slack length of sarcomeres and cardiac myocytes was also measured (cells are slack when they are relaxed and do not experience external forces). The slack sarcomere length in $\mathrm{Ttn}^{\Delta \mathrm{C}-2}$ myocytes was reduced by $170 \mathrm{~nm}$ (Fig. $4 \mathrm{a}$, left), findings that are supported by the $174 \mathrm{~nm}$ reduced slack sarcomere length in papillary muscles (Fig. 4a, right). The shorter slack sarcomere length is in contrast to the longer slack length of the myocyte (Fig. 4b), indicating the number of serially linked sarcomeres is increased in $T t n^{\Delta C l-2}$ myocytes, based on the measured mean cell length and sarcomere length by $13 \%$. The reduced slack sarcomere length of $T t n^{\Delta C 1-2}$ myocytes is also expected to give rise to higher passive tensions when passive cells are stretched to

\section{Table 2 Pressure (P)-volume (V) analysis of the left} ventricular chamber of the heart

\begin{tabular}{|c|c|c|c|}
\hline & WT & $T t n^{\Delta C 1-2}$ & $p$-value \\
\hline$n$ & 7 & 8 & \\
\hline HR (b.p.m.) & $497 \pm 6$ & $463 \pm 6$ & 0.0015 \\
\hline $\mathrm{ESP}(\mathrm{mm} \mathrm{Hg})$ & $86.8 \pm 2.8$ & $66.7 \pm 3.2$ & 0.0004 \\
\hline $\mathrm{EDP}(\mathrm{mm} \mathrm{Hg})$ & $2.5 \pm 0.5$ & $2.6 \pm 0.7$ & 0.955 \\
\hline $\mathrm{dP} / \mathrm{dt} \max (\mathrm{mm} \mathrm{Hg} / \mathrm{s})$ & $8460 \pm 302$ & $5591 \pm 200$ & $<0.0001$ \\
\hline $\mathrm{dP} / \mathrm{dt} \min (\mathrm{mm} \mathrm{Hg} / \mathrm{s})$ & $-8319 \pm 357$ & $-3842 \pm 227$ & $<0.0001$ \\
\hline $\operatorname{ESV}(\mu \mathrm{L})$ & $19.4 \pm \overline{3} .1$ & $43.6 \pm \overline{5} .3$ & 0.002 \\
\hline $\operatorname{EDV}(\mu \mathrm{L})$ & $59.5 \pm 4.6$ & $84.4 \pm 5.0$ & 0.003 \\
\hline$S V(\mu L)$ & $40.1 \pm 3.9$ & $40.8 \pm 2.6$ & 0.88 \\
\hline $\mathrm{CO}(\mathrm{mL} / \mathrm{min})$ & $19.9 \pm 1.8$ & $18.9 \pm 1.3$ & 0.656 \\
\hline $\mathrm{EF}(\%)$ & $67.9 \pm 4.2$ & $49.2 \pm 4.1$ & 0.007 \\
\hline $\mathrm{EA}(\mathrm{mm} \mathrm{Hg} / \mu \mathrm{L})$ & $2.23 \pm 0.17$ & $1.72 \pm 0.17$ & 0.057 \\
\hline Tau Glantz (ms) & $9.4 \pm 0.5$ & $22.7 \pm 2.4$ & 0.0002 \\
\hline PRSW & $81.7 \pm 3.5$ & $45.3 \pm 2.4$ & $p<0.0001$ \\
\hline ESPVR (ES) & $3.6 \pm 0.3$ & $1.6 \pm 0.2$ & $p<0.0001$ \\
\hline ESPVR (VO) & $-4.9 \pm 1.6$ & $-10.9 \pm 4.1$ & 0.667 \\
\hline EDPVR $(\beta)$ & $0.031 \pm 0.007$ & $0.037 \pm 0.008$ & 0.584 \\
\hline Arterioventr. coupling (EA/ES) & $0.6 \pm 0.1$ & $1.2 \pm 0.2$ & 0.039 \\
\hline
\end{tabular}

HR: heart rate; ESP: end-systolic pressure; EDP: end-diastolic pressure; $\mathrm{dP} / \mathrm{dt}$ max: maximal rate of pressure development; $\mathrm{dP} / \mathrm{dt}$ min: maximal rate of pressure reduction; ESV: end-systolic volume; EDV: end-diastolic volume; SV: stroke volume; EF: ejection fraction; EA, effective arterial elastance; Tau Glantz: left ventricular relaxation time constant; PRSW: preload recruitable stroke work; ESPVR (ES): slope of end-systolic pressure-volume relation; ESPVR (VO): volume intercept of end-systolic pressure-volume relation; $\operatorname{EDPVR}(\beta)$ : exponent of exponential fit of end-diastolic pressure-volume relation.

Male mice at 62-74 days of age were used $\left(n=7 \mathrm{WT}\right.$ and $\left.n=8 T t n^{\Delta C 1-2}\right)$. Mean values \pm s.e.m. Male mice at $62-74$ days of age were used $\left(n=7 \mathrm{WT}\right.$ and $n=8 \mathrm{Tt} n^{\Delta(-2)}$. Mean values \pm s.e.m.
are shown. Statistical analysis with a two-tailed $t$-test. Additional statistical analysis is provided in Supplementary Table 4 a given sarcomere length, consistent with the measured passive tension values (Fig. 4c). The passive tension curves of $\mathrm{Ttn}^{\Delta \mathrm{Cl} 1-2}$ and WT myocytes are similar in shape but shifted along the sarcomere length axis. Right shifting the $T t n^{\Delta C 1-2}$ curve by $173 \mathrm{~nm}$ (the difference in A-band width) reproduces the WT curve (see the dashed red curve in Fig. 4c).

\section{Discussion}

The thick filament of vertebrate muscle is of a precisely controlled length, and in the present work we addressed the mechanism by which thick filament length regulation is achieved. Ever since the discovery that titin runs along the thick filament length ${ }^{9}$, and that it contains super-repeats that span a distance that coincides with the $\sim 43 \mathrm{~nm}$ myosin helical repeat of the thick filament ${ }^{20}$, it has been speculated that titin functions as a thick filament length ruler $^{5-8}$. To test the role of titin in thick filament length regulation we created the $T t n^{\Delta C 1-2}$ mouse in which two of titin's superrepeats (C1 and $\mathrm{C} 2$ ) were deleted. Structural studies in both cardiac and skeletal muscles reveal that deleting two super-repeats from the A-band segment of titin reduces thick filament length with each of titin's C-zone repeats being responsible for a quantal $43 \mathrm{~nm}$ thick filament length. Below we discuss the structural and functional findings of this work in detail.

To test the titin ruler hypothesis a mouse model was created in which two of titin's super-repeats were genetically deleted. Although differently sized deletions could have been made, the titin gene structure limited the available choices as we considered it important to delete an integral number of super-repeats, required introns of sufficiently large size in order to place selection markers, and we had to avoid disrupting the reading frame. Hence, a deletion of two super-repeats was one of our best options. Indeed, an internally shortened mutant titin protein is made by the $T t n^{\Delta C 1-2}$ mouse that is normally incorporated in the sarcomere (Fig. 1c, d). The deletion is compatible with life, as revealed by breeding heterozygous $T t n^{\Delta C 1-2}$ parents that produce both Het and Hom mice at Mendelian ratios and with normal body and muscle weights (Supplementary Fig. 1b-d). A concern when targeting the TTN gene is inadvertently causing alterations in splicing of the remaining exons, but the RNAseq-based exon map did not reveal changes in exon usage in LV myocardium (Fig. 1b), indicating that no major changes in splicing had occurred. However, at the protein level small but significant differences in isoform expression were found: the N2BA titin isoform was upregulated relative to the $\mathrm{N} 2 \mathrm{~B}$ isoform
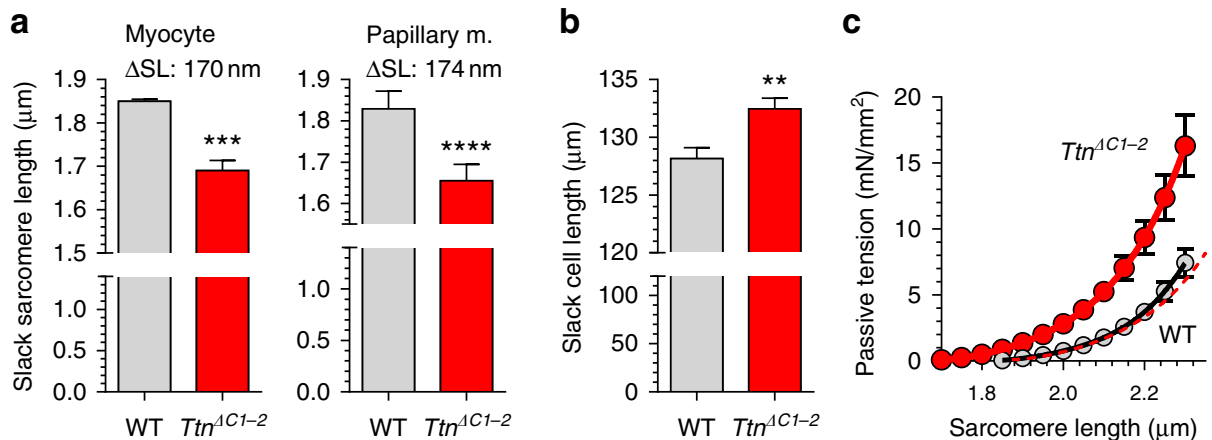

Fig. 4 Effect of reduced thick filament length on slack sarcomere length (a), slack cell length (b), and passive tension (c). Skinned myocytes (a, left) and skinned papillary muscle (a, right) isolated from the left ventricle have shorter slack sarcomere length in $\mathrm{Ttn}^{\Delta \mathrm{Cl}-2}$ mice, but cell length is longer (b). c Passive tension of skinned cardiac myocytes as a function of sarcomere length increases steeper in Ttn ${ }^{\Delta C 1-2}$ mice. Individual data points were fit with a fourth-order polynomial function. Dashed red line represents the fit of the $T t_{n} \Delta{ }^{C 1-2}$ data right-shifted by $173 \mathrm{~nm}$. Note that the right-shifted $T t_{n} \Delta \mathrm{Cl}-2$ fit overlaps well with WT data. a, left: mean results from 5 hearts (20 cells per heart); a, right: mean results from 12 muscles from 12 mice; c mean results from 5 myocytes from 3 hearts. Female mice at 48-56 days of age were used $\left(n=6\right.$ WT and $\left.n=6 T t n^{\Delta C 1-2}\right)$. Error bars are s.e.m. Statistical analysis with a two-tailed $t$-test. Additional statistical analysis is provided in Supplementary Table 4 ( ${ }^{\star \star}$ denotes $p<0.01,{ }^{\star \star \star} p<0.001$, and ${ }^{\star \star \star \star} p<0.0001$ ) 
(Supplementary Fig. 2A). This combined with the absence of alterations in titin at the transcript level suggests changes in protein turnover, i.e., a slowdown in degradation of the N2BA isoform and an increase in degradation of the N2B isoform. These two isoforms have the same exon composition in the A-band and Z-disk regions of the molecule but differ in their I-band regions ${ }^{33}, 34$. The I-band region of the N2BA titin molecule has, compared to $\mathrm{N} 2 \mathrm{~B}$ titin, additional exons spliced in, and is therefore more compliant (i.e., it generates less force for a given extension $)^{35,36}$. The upregulation of N2BA titin that was found in the $T t n^{\Delta C 1-2}$ mouse can be viewed as a beneficial adaptation that offsets the increased passive stiffness that is expected from the shortened A-band in the $T t n^{\Delta C 1-2}$ mouse (see below).

Determining the thick filament length from TEM images requires a correction for the shrinkage that is well known to take place during sample preparation ${ }^{3}$. Large levels of A-band shrinkage were found in our study in both cardiac and skeletal muscles, with a degree of shrinkage that was $\sim 20-30 \%$ in short sarcomeres (Supplementary Figs. 4 and 5). As sarcomere length was increased, the degree of shrinkage was reduced (in sarcomere $>\sim 2.5 \mu \mathrm{m}$ to $5-10 \%$ shrinkage), explaining the large slopes of the regression lines fit to the A-band width versus sarcomere length data (Supplementary Fig. 3). Correcting for shrinkage largely abolishes this sarcomere length dependence and results in a mean thick filament length in WT mice of $1.61 \mu \mathrm{m}$ in cardiac and 1.58 $\mu \mathrm{m}$ in skeletal muscle (Table 1 ). These values are in line with the $1.62 \mu \mathrm{m}$ reported by Huxley and colleagues ${ }^{3}$, using a specialized rapid freeze/freeze substitution technique, indicating that the shrinkage correction methods used in our work are valid. The explanation for the finding that the level of shrinkage is reduced as sarcomere length is increased and that shrinkage is less in the $T t n^{\Delta C 1-2}$ model (Supplementary Figs. $4 \mathrm{~B}$ and $5 \mathrm{D}$ ) is unclear. We speculate that the titin-based force that pulls outward on the thick filaments counters shrinkage, as this force increases with sarcomere length and is higher in $\mathrm{Ttn}^{\Delta C 1-2}$ mice (Fig. 4c). It is also worthwhile noting that the $\mathrm{CMyBP}-\mathrm{C}$ antibody that was used to study shrinkage revealed in myocardium of WT mice 9 cMyBP-C stripes (consistent with findings by others ${ }^{29}, 37$ ) but in Ttn $^{\Delta C 1-2}$ mice the outer stripe (stripe 9) was absent (with occasional evidence for spotty labeling). This suggests that titin super-repeat 1 must be different from the others, and is not involved in anchoring cMyBP-C, but that super-repeat 2 is. This is unexpected since many of the thick filament models locate cMyBP-C on titin super-repeats $1-9^{29}$, warranting follow-up studies on the cMyBP-C-binding properties of the terminal titin super-repeats.

Importantly, following shrinkage correction the thick filament length is reduced in $T t n^{\Delta C 1-2}$ mice by $173 \mathrm{~nm}$ in cardiac muscle and $168 \mathrm{~nm}$ in skeletal muscle (Table 1). Considering that each half thick filament is associated with a titin molecule, the thick filament length reductions that we obtained are in line with the concept of a $2 \times 43 \mathrm{~nm}$ shortened titin ruler. Thus, for the first time highly supportive evidence exists for titin as a molecular ruler. To explain the underlying mechanism, at least two possibilities present themselves. First, a sequence $\mathrm{N}$ terminal to the deleted C-zones might determine the thick filament length and its distance to the M-band is set by the combined length of the $\mathrm{C}$ zone repeats ${ }^{8}$. Because eliminating the I/A junction of titin does not alter thick filament length ${ }^{19}$, its role is unlikely, but a role for the D-zone of titin cannot be ruled out as of yet. Second, the Czone repeats might play a more direct role in thick filament length regulation, as suggested by the interactions between the Fn domains of the C-zone super-repeats and myosin ${ }^{21}$ and consistent with the recently proposed hypothesis that titin stabilizes the thick filament ${ }^{7}$. Considering that it is likely that there are 6 titin molecules that run along the thick filament ${ }^{38}$, spread around its periphery ${ }^{1}$, we speculate that this interaction between C-zone repeats and myosin holds the thick filament in a 'cage' (akin to a Chinese finger trap) that stabilizes the thick filament and prevents depolymerization.

A shortened thick filament is predicted to have multiple functional effects, including a reduction in the slack sarcomere length. The slack sarcomere length is determined by the end-toend length of titin's I-band region at which titin exerts no force, a length that is expected to be the same in WT and Ttn ${ }^{\Delta C 1-2}$ myocytes, plus the length of the thick filament. Thus, the shortened thick filament in $T t n^{\Delta C 1-2}$ myocytes/muscles is predicted to lower the slack sarcomere length by $\sim 170 \mathrm{~nm}$, which is consistent with the measured values (Fig. 4a). The reduced slack sarcomere length in the $T t n^{\Delta C 1-2}$ myocytes is expected to increase the number of serially linked titin molecules per unit muscle length by $\sim 9 \%$ (reduction in slack sarcomere length $(170 \mathrm{~nm})$ divided by the slack sarcomere length $(\sim 1850 \mathrm{~nm}))$. This increased number of titin molecules is likely to explain why the reduced size of titin in the $T n^{\Delta C 1-2}$ mouse does not result in the expected $\sim 8 \%$ reduction in the total level of titin protein (the deletion of the two super-repeats, $239.5 \mathrm{kDa}$, divided by the Mw of WT N2B titin, $\sim 3000 \mathrm{kDa}$ ) (Supplementary Fig. 2B). Thus, despite the titin molecule is smaller, the total titin protein level will not be reduced, because the number of serially linked titin molecules per unit length of muscle is increased. A similar argument can be made for myosin, the expression level of which is expected to be reduced by $\sim 11 \%$ in the $T t n^{\Delta C 1-2}$ mouse (length reduction of thick filament $(\sim 170 \mathrm{~nm})$ divided by thick filament length $(\sim 1600$ $\mathrm{nm})$ ) yet the measured expression level shows no differences (Supplementary Fig. 2B), i.e., the reduced slack sarcomere length increases the number of thick filaments per unit muscle length. As expected, the shorter slack sarcomere length also increases the level of passive tension in stretched cardiac myocytes of $T t n^{\Delta C 1-2}$ mice, relative to that of WT mice (Fig. 4c). However, what is unexpected is that this is not reflected in the diastolic chamber stiffness (the $\beta$-coefficient of the EDPVR is unaltered, Table 2). This contrasts previous work in which titin was genetically altered to either increase titin-based tension ${ }^{19}, 39,40$ or lower titin-based tension $^{41,42}$ and LV diastolic chamber stiffness was found to be altered accordingly, findings that have led to the view that titin is a dominant contributor to diastolic chamber stiffness. A possible explanation for the discordance between the results of cardiac myocytes and that of the LV chamber in the present work is a sarcomere length operating range that is shifted to shorter sarcomere lengths in $T t n^{\Delta C 1-2}$ mice compared to WT mice, i.e., a shift of $\sim 170 \mathrm{~nm}$ would result in an identical titin-based passive tension of the myocytes and thus an unaltered chamber stiffness. In summary, shortening the thick filaments reduces the slack sarcomere length and increases the passive tension in stretched myocytes, but this does not cause an increase in diastolic chamber stiffness in $T t n^{\Delta C 1-2}$ mice, likely because the sarcomere length range during the cardiac cycle is shifted to shorter sarcomere lengths.

Active tension measurements revealed that skeletal muscles of $T t n^{\Delta C 1-2}$ mice generate less force and have a steeper descending limb of their force-sarcomere length relation. Additionally, the heart generates less systolic pressure and has reductions in the slope of the ESPVR and the PRSW, both indexes of myocardial contractility ${ }^{31}$. Measurements in cardiac myocytes show that calcium transients are unaltered indicating that the depressed contractility of $\mathrm{Ttn}^{\Delta \mathrm{Cl}-2}$ mice is not due to a reduction in activating calcium levels. Instead, the depressed contractility is consistent with the shorter thick filaments that our structural studies revealed, as fewer force generating myosin molecules in the shortened thick filament will limit the active force level that can be attained. Thus, the depressed contractility of skeletal and cardiac muscles in $\mathrm{Ttn}^{\Delta \mathrm{Cl}-2}$ mice shows that thick filament 
length regulation is functionally important for optimal force generation.

Compared to WT mice, the LV chamber of $T t n^{\Delta C 1-2}$ mice has a dilated phenotype, as revealed by the increased diastolic LV chamber dimension, the decreased LV wall thickness, and the increased eccentricity index (Supplementary Table 1), and supported by the elongation of the cardiac myocytes of $T_{t n}{ }^{\Delta C 1-2}$ mice (Fig. 4c). In the recent study by Davis et al. ${ }^{43}$, a mathematical model of hypertrophic versus dilated cardiomyopathy was proposed in which the level of myofilament tension is critical in the growth decision that myocytes make with a reduction in tension resulting in eccentric growth and a dilated phenotype. Our finding of a dilated phenotype in the $T t n^{\Delta C 1-2}$ mouse that has a reduced tension due to shortened thick filaments supports this model. The underlying mechanism is unclear but analogous to what has been reported in other DCM models ${ }^{43}$ that impaired MEK1/ERK signaling might be involved and should be examined in future work, including the possible role of titin's I-band region that is known to interact with components of this signaling pathway $^{44}$. Finally, several recent landmark next-generation sequencing studies revealed that truncation variants in the titin gene (TTNtv) are prevalent in DCM patients ${ }^{15-18,27,45}$. Interestingly, most of the TTNtvs are found in the A-band segment of titin (including 12 within the $\mathrm{C} 1$ and $\mathrm{C} 2$ repeats) but how they cause DCM is unknown. We propose that these truncation mutations impact titin's role in thick filament length regulation and that this causes a force reduction that triggers growth decisions that lead to DCM.

In summary, our studies reveal for the first time the important role of titin in regulating thick filament length, with each of titin's C-zone repeats being responsible for a quantal $43 \mathrm{~nm}$ thick filament length. We conclude that thick filament length regulation is titin based and that this mechanism is crucial for maintaining muscle health.

\section{Methods}

Generation of the $\mathbf{T t n}^{\Delta \mathbf{C 1}-\mathbf{2}}$ mouse model. All experiments in this study were conducted in accordance with the National Institutes of Health (NIH) Guide for the Care and Use of Laboratory Animals, and all protocols were approved by University of Arizona's Institutional Animal Care and Use Committee. The $T t n^{\Delta C 1-2}$ mice were produced via homologous recombination by the Genetically Engineered Mouse Model Core (University of Arizona, Tucson, AZ, USA). The targeting vector was assembled using PCR from C57BL/6J genomic DNA (Jackson Laboratory) into a vector which includes an FRT-flanked neoR selection cassette. After transformation by electroporation and G418 selection of 129S6 ES cells the targeted clones were identified and injected into C57BL/6J blastocysts. Germline transmission was recovered from 1 chimera (verified by PCR). The neoR cassette was removed by mating to ActinB:FLPe mice (Jackson), offsprings were then backcrossed to C57BL/6J. The deleted genomic DNA corresponds to an internal deletion of 2177 amino acids $(239.5 \mathrm{kDa})$.

Body weight analysis and muscle dissection. Body weight data from WT (18 males, 14 females), $\operatorname{Ttn}^{\Delta C 1-2}$ (17 males, 10 females), and Het (30 males, 22 females) mice were collected from 16 days to 3 months after birth. Dissection of muscles was performed on heparinized mice (10 U/g, USP, Hospira Inc.) anesthetized with isoflurane (USP, Phoenix Pharmaceuticals, Inc.) and killed by cervical dislocation. The hearts were removed, and both atria and the right ventricle were rapidly dissected and weighed. LV papillary muscles were dissected and skinned in relaxing solution ((in mM): $40 \mathrm{BES}, 10 \mathrm{EGTA}, 6.56 \mathrm{MgCl}_{2}, 5.88 \mathrm{Na}$-ATP, 1 dithiothreitol (DTT), $46.35 \mathrm{~K}$-propionate, 15 creatine phosphate, $\mathrm{pH} 7.0)$ containing $1 \%(\mathrm{w} / \mathrm{v})$ Triton X-100 and protease inhibitors ((in $\mathrm{mM})$ : 0.1 E64, 0.47 leupeptin and 0.25 phenylmethylsulfonyl fluoride (PMSF)). The following skeletal muscles were dissected and rapidly weighed: $\mathrm{m}$. tibialis cranialis, $\mathrm{m}$. EDL, $\mathrm{m}$. soleus, $\mathrm{m}$. plantaris, $\mathrm{m}$. gastrocnemius, and $\mathrm{m}$. quadriceps. Both tibias were removed, and the mean tibia length was used for normalization of muscle weight data. Papillary and EDL muscles were skinned overnight at $4{ }^{\circ} \mathrm{C}$ in relaxing solution containing $1 \%$ Triton $\mathrm{X}-100$ and protease inhibitors, washed thoroughly with relaxing solution, and stored in $50 \%$ glycerol/relaxing solution at $-20^{\circ} \mathrm{C}$ and used within 2 weeks for experiments. All other muscles were quick frozen in liquid nitrogen and stored at $-80^{\circ} \mathrm{C}$.
Titin exon usage. Total RNA was isolated from LV apex of 2-month-old WT and $T t n^{\Delta C 1-2}$ male $(n=6$ each) mice using the RNeasy Fibrous Tissue Mini Kit (Qiagen). A Bioanalyzer (Agilent) was used to verify RNA quality and integrity and to determine concentration. Three samples per genotype were prepared with equal amounts of total RNA from two mice per sample. Library preparation and RNAseq were performed by the University of Chicago Genomics Facility following Illumina protocols for RiboZero depletion, TruSeq single-stranded total RNA library construction, and sequencing $(2 \times 100 \mathrm{bp}$ paired-end reads) on the HiSEQ4000. The sequencing depth was sufficient to yield robust transcript level measurements with a per-sample mean of $40.0 \pm 0.5 \times 10^{6}$ paired-end reads aligned to the GRC38v11/ mm10 Mus musculus reference genome. Analysis was performed on the Illumina BaseSpace cloud platform, the RNAExpress app was run which uses an analysis pipeline with the STAR short read aligner (which maps reads based on the GRC38/ $\mathrm{mm} 10$ genome including identification of splice sites used) ${ }^{46}$ which outputs counts for splice junction usage; counts for the titin region on chromosome 2 were extracted to produce Fig. $1 \mathrm{~b}$.

Quantification of protein expression. Flash frozen LV and EDL tissues were pulverized in liquid nitrogen and then solubilized in urea buffer ((in mol/L): 8 urea, 2 thiourea, 0.05 tris- $\mathrm{HCl}, 0.075$ dithiothreitol with $3 \%$ SDS and $0.03 \%$ bromophenol blue, $\mathrm{pH} 6.8$ ) and $50 \%$ glycerol with protease inhibitors ((in $\mathrm{mmol} / \mathrm{L}$ ): 0.04 E64, 0.16 leupeptin and $0.2 \mathrm{PMSF}$ ) at $60^{\circ} \mathrm{C}$ for $10 \mathrm{~min}^{47}$. Samples were centrifuged at 13,000 RPM for $5 \mathrm{~min}$, aliquoted, flash frozen in liquid nitrogen, and stored at $-80^{\circ} \mathrm{C}$. Titin isoform analysis was performed on solubilized samples (from $n=7$ male and 3 female WT; $n=7$ male and 2 female Tt $n^{\Delta C 1-2} 58-75$-day-old mice) using a vertical SDS-agarose gel system as previously described ${ }^{48}$. Then, $1 \%$ gels were run at $15 \mathrm{~mA}$ per gel for 3:20, then stained using Coomassie brilliant blue, scanned using a commercial scanner, and analyzed with One-D scan (Scanalytics Inc.). The integrated optical density (IOD) of titin and major histocompatibility complex (MHC) were determined as a function of loading volume (in a range of 5 volumes). The slope of the linear relationship between IOD and loading was obtained for each protein to quantify expression ratios. For western blotting, solubilized samples were run on a $0.8 \%$ agarose gel, then transferred onto polyvinylidene difluoride membranes using a semi-dry transfer unit (Trans-Blot Cell, Bio-Rad). Blots were stained with Ponceau $S$ to visualize the total protein transferred. Blots were then probed with primary antibodies followed by secondary antibodies conjugated with infrared fluorescent dyes. Blots were scanned using an Odyssey Infrared Imaging System (Li-COR Biosciences). The primary antibodies that were used for western blotting, superresolution SIM, and IEM are shown in Supplementary Table 3. Uncropped western blot images using Z1Z2 antibody (Supplementary Fig. 7) and M8-9 antibody (Supplementary Fig. 8) are provided. For MHC protein expression, $n=8 \mathrm{WT}$ and $n=8 T t n^{\Delta C 1-2} 58$-75-day-old mice (6 males and 2 females in each group) were used.

Superresolution SIM (SR-SIM). WT $(n=10)$ and $\operatorname{Ttn}^{\Delta C 1-2}(n=9)$ male mice aged 65 days were used in the SR-SIM study. Skinned cardiac and EDL myofibril bundles were embedded in optimal cutting temperature (OCT) compound and immediately frozen in 2-methylbutane precooled in liquid nitrogen. Then, $5 \mu \mathrm{m}$ thick cryosections were cut and mounted onto microscope slides. Tissue sections were permeabilized in $0.2 \%$ Triton X-100/phosphate-buffered saline (PBS) for $20 \mathrm{~min}$ at room temperature, blocked with $2 \%$ bovine serum albumin (BSA) and $1 \%$ normal donkey serum in PBS for $1 \mathrm{~h}$ at $4{ }^{\circ} \mathrm{C}$, and incubated overnight at $4{ }^{\circ} \mathrm{C}$ with primary antibodies diluted in blocking solution. The primary antibodies included (Supplementary Table 3): a rabbit polyclonal anti-Titin I103 $(3.33 \mu \mathrm{g} / \mathrm{mL})$, a mouse monoclonal anti-Titin T12 $(2.5 \mu \mathrm{g} / \mathrm{mL})$ (Boehringer Mannheim), a mouse monoclonal anti- $\alpha$-actinin (1:4000) (EA-53, Sigma-Aldrich) antibody, and a guinea pig anti-Titin A165 antiserum (enzyme-linked immunosorbent assay titer: $>1: 200,000$, dilution: 1:50). Sections were then washed with PBS for $2 \times 30 \mathrm{~min}$ and incubated with secondary antibodies diluted in PBS for $3 \mathrm{~h}$ at room temperature. The secondary antibodies (dilution: 1:500), obtained from Invitrogen and Abcam, included: AlexaFluor-488 conjugated goat anti-mouse IgG, AlexaFluor-568 conjugated goat anti-rabbit IgG, AlexaFluor-568 conjugated goat anti-guinea pig IgG, and AlexaFluor-647 conjugated donkey anti-rabbit IgG. The sections were then washed with PBS for $2 \times 15 \mathrm{~min}$ and covered with number $1.5 \mathrm{H}$ coverslips using ProLong Diamond (Thermo Scientific, Inc.). A Zeiss ELYRA S1 SR-SIM microscope was used with ultraviolet and solid-state laser $(488 / 561 / 642 \mathrm{~nm})$ illumination sources, a $100 \times$ oil immersion objective $(\mathrm{NA}=1.46)$, and a sCMOS camera. Typical imaging was performed on a $49.34 \times 49.34 \mu \mathrm{m}^{2}$ area with $1280 \times 1280$ pixel dimensions. Image stacks comprising of 30 slices were acquired with $0.101 \mu \mathrm{m} \mathrm{Z}$ steps, five angles, and five phases/angle for each slice. Image reconstruction and fluorescence intensity plot profile generation were performed with ZEN 2 software (Zeiss). Plot profiles were fit with Gaussian curves to determine the epitope peak position using Fityk 1.3.0 software. A-band width was determined from the I103 epitope positions across the Z-disk. The A165 epitope locations used for shrinkage correction were measured across the A-band.

TEM and IEM. Skinned LV papillary muscle and EDL skeletal muscle of $~ 8$-weekold male homozygous $\operatorname{Ttn}^{\Delta C 1-2}$ mice $(n=6)$ and littermate WT controls $(n=6)$ were stretched from the slack length at different degrees ( 20\% to $50 \%)$ and 
processed for TEM. Briefly, fixation of muscle tissue was performed with $3 \%$ paraformaldehyde in $10 \mathrm{mM}$ PBS, $\mathrm{pH} 7.2$, for $30 \mathrm{~min}$ at $4{ }^{\circ} \mathrm{C}$. This was followed by a secondary fixation with $3 \%$ glutaraldehyde and $0.02 \%$ tannic acid ${ }^{49}$ in the same buffer, and postfixed in $1 \% \mathrm{OsO}_{4}$ in $\mathrm{PBS}$ for $30 \mathrm{~min}$ at $4{ }^{\circ} \mathrm{C}$. Samples were then dehydrated in an ethanol graded series, infiltrated with propylene oxide, and transferred to a mix of 1:1 propylene oxide/Araldite 502/Embed 812 (Epon-812, EMS). Subsequently, samples were transferred to a pure Araldite 502/Embed 812 resin and polymerized for $48 \mathrm{~h}$ at $60^{\circ} \mathrm{C}$. Ultrathin, $60 \mathrm{~nm}$ longitudinal sections were obtained with a Reichert-Jung ultramicrotome and contrasted with $1 \%$ potassium permanganate $e^{50}$ and lead citrate. Samples were observed in a TECNAI Spirit G2 transmission electron microscope (FEI) and images acquired with a sidemounted AMT Image Capture Engine V6.02 (4Mpix) digital camera operated at $100 \mathrm{kV}$. Digital images were stored for A-band width measurements with ImageJ $1.49 \mathrm{v}$ (NIH, USA).

Ultrastructural immunolabeling of I103 titin, cMyBP-C (C5-C7), and A77-8 (C-zone 4) epitopes was performed on skinned cardiac and skeletal muscle from WT mice $(n=9)$ and $\operatorname{Ttn}^{\Delta C 1-2}$ mice $(n=9)$ stretched to different degrees from the slack length by the preembedding technique with modifications ${ }^{51}$. Fiber bundles from LV muscle and EDL skeletal muscle were skinned twice and washed in relaxing solution as for TEM, previous to fixation with 3\% paraformaldehyde in PBS for $30 \mathrm{~min}$ at $4{ }^{\circ} \mathrm{C}$, followed by washes with PBS, and with PBS containing protease inhibitors. Blocking was performed with different concentrations of BSA $(1 \%, 0.5 \%, 0.25 \%)$ in PBS containing protease inhibitors and $0.05 \%$ Tween-20, according to the used primary antibodies (Supplementary Table 3) from rabbit: anti-I103 $(1: 25,24 \mathrm{~h})$, anti-cMyBP-C $(1: 8,72 \mathrm{~h})$, and anti-A77-8 (GenScript, 1:20, $48 \mathrm{~h}$ ), respectively. After rinsing with PBS containing protease inhibitors, muscles were incubated with the secondary antibodies Fab goat anti-rabbit antibody IgG (AP 132, Millipore, 1:25) or AlexaFluor-568 goat anti-rabbit IgG (ab175471, Invitrogen, 1:30) as appropriated. All incubations were performed in a humid chamber at $4{ }^{\circ} \mathrm{C}$. Labeling of cardiac muscle with cMyBP-C was performed on bundle fibers untreated and treated with 1:2 of relaxing solution/gelsolin (a recombinant $45 \mathrm{kDa} \mathrm{N}$-terminal fragment that is calcium independent $\mathrm{t}^{52}, 0.6 \mathrm{mg} /$ $\mathrm{mL}$ (in $\mathrm{mM}$ ): 25 MOPS, $150 \mathrm{KCl}, 1 \mathrm{DTT}, 1$ EGTA, pH 7.4) with continuous shaking for $1 \mathrm{~h}$ at $4{ }^{\circ} \mathrm{C}$ to extract thin filaments, previous to the fixation step. Thin filament extraction was used in the experiments on cardiac muscle to make it easier for the $\mathrm{cMyBP}-\mathrm{C}$ antibody to reach its binding site and, additionally, to enhance the contrast between A-bands and I-bands, making it easier to measure thick filament length (e.g., Fig. 2b and Supplementary Fig. 4A). Skeletal muscle fibers were hard to extract, confirming previous findings ${ }^{53}$ and were therefore studied with thin filaments in place (e.g. Fig. $2 \mathrm{c}$ and Supplementary Fig. 5A). To test whether thin-filament extraction affects the results we also performed experiments on cardiac muscle that were not gelsolin treated. We obtained results that were indistinguishable from fibers that had been gelsolin treated. After labeling, muscle tissues were washed in PBS and fixed with $3 \%$ glutaraldehyde in the same buffer, then processed for TEM and images recorded as explained above. Digital images were calibrated with ImageJ and the density plot profiles were analyzed to determine the width of A-band from the following measurements: I103 titin epitope-to-epitope distance on cardiac and skeletal muscle, the spacing between cMyBP-C-labeled stripes on cardiac muscle, and the A77-8 epitope-to-epitope distance on skeletal muscle across the M-band. These measurements were used to determine the degree of A-band shrinkage to convert measured A-band into shrinkage-corrected A-band width values, and then analyzed with linear regression to test differences from slopes linear fits and line offsets.

In vivo pressure-volume measurements. An in vivo $\mathrm{PV}$ analysis was performed on 62-74-day-old male mice ( $n=7 \mathrm{WT}$ and $n=8 \mathrm{Ttn}^{\Delta C 1-2}$ ) using an Advantage Admittance Derived Volume Measurement System (SciSense, Inc.) and 1.2 F catheters with $4.5 \mathrm{~mm}$ electrode spacing. Mice were anesthetized and ventilated with $3 \%$ isoflurane for induction and $1.5 \%$ for maintenance with body temperature maintained at $37^{\circ} \mathrm{C}$. (Note that the level of sedation varies slightly among animals and that therefore we will not assign importance to small differences in heart rates between groups.) Anesthetized mice were secured and a bilateral subcostal incision was made. The diaphragm was opened to expose the heart. The catheter was inserted into the LV via apical approach. The inferior vena cava was located and occluded during a sigh (pause) in ventilation to acquire load-independent indexes. Data acquisition and analysis were performed in LabScribe2 (iWorx). EDPVR was analyzed using a monoexponential fit $\left(P=C+A e^{\beta V}\right)$ with the exponent $(\beta)$ reported as the stiffness ${ }^{31}$. Details are in Supplementary Table 1.

Mouse echocardiography. Male WT $(n=8)$ and $\operatorname{Ttn}^{\Delta C l-2}(n=8)$ mice at 50 days of age were anesthetized with $1 \%$ isoflurane, then placed in dorsal recumbence on a heated platform (body temperature: $37^{\circ} \mathrm{C}$ ). Transthoracic echo images were obtained with a Vevo 2100 High-Resolution Imaging System (Visual-Sonics, Inc.) using the model MS550D scan head for cardiac imaging and MS250 to measure aortic flow velocity. Care was taken to avoid animal contact and excessive pressure which could induce bradycardia. Imaging was performed at a depth setting of $1 \mathrm{~cm}$. Images were collected and stored as a digital cine loop for offline calculations. Standard imaging planes and functional calculations were obtained according to American Society of Echocardiography guidelines. The parasternal long-axis view and mid-wall cross-sectional view of the LV were used to guide calculations of percentage fractional shortening, percentage ejection fraction, and ventricular dimensions and volumes. The left atrial dimension was measured in the long-axis view directly below the aortic valve leaflets. Passive LV filling peak velocity, $E(\mathrm{~cm} /$ $\mathrm{s})$, and atrial contraction flow peak velocity, $A(\mathrm{~cm} / \mathrm{s})$, were acquired from the images of mitral valve Doppler flow from tilted parasternal long-axis views. A sweep speed of $100 \mathrm{~mm} / \mathrm{s}$ was used for M-mode and Doppler studies. The heart rates of animals during the echocardiographic study were maintained in the range of 500-550 beats/min for M-mode, $450-500$ beats $/ \mathrm{min}$ for B-mode, and $350-450$ beats/min for Doppler studies. For details see Supplementary Table 2.

Cell isolation and calcium transients. The 48-56-day-old female mice (6 WT and $6 T^{\Delta} n^{\Delta C 1-2}$ ) were heparinized and killed by cervical dislocation under isoflurane. The heart was removed and cannulated via the aorta for retrograde coronary perfusion ${ }^{54}$. The heart was perfused for $4 \mathrm{~min}$ with perfusion buffer, followed by 20 min digestion using $0.05 \mathrm{mg} / \mathrm{mL}$ Liberase $\mathrm{TM}$ and $13 \mu \mathrm{M} \mathrm{CaCl}_{2}$ in perfusion buffer. Digestion was halted by placing the heart in myocyte stopping buffer $\left(0.08 \mathrm{mg} / \mathrm{mL}\right.$ bovine calf serum and $8 \mu \mathrm{M} \mathrm{CaCl}_{2}$ in perfusion buffer with protease inhibitors). The LV was cut into small pieces, triturated several times with a transfer pipette, and then filtered through a $300 \mu \mathrm{m}$ nylon mesh filter to acquire intact cardiomyocytes. $\mathrm{Ca}^{2+}$ ( $1 \mathrm{mM}$ final concentration) was reintroduced to the cardiomyocyte suspension. Isolated LV cardiomyocytes were incubated for $10 \mathrm{~min}$ at room temperature with $2 \mu \mathrm{M}$ Fura-2 AM (F-1225, Life Technologies, Inc.) in perfusion buffer containing $0.08 \mathrm{mg} / \mathrm{mL}$ bovine calf serum and $1 \mathrm{mM} \mathrm{CaCl} 2$ and resuspended in $1.8 \mathrm{mM} \mathrm{Ca}^{2+}$ Dulbecco's modified Eagle's medium (DMEM)/F-12. All intact cell experiments were performed at $37^{\circ} \mathrm{C}$ in DMEM/F-12 plus $10 \mu \mathrm{g} / \mathrm{mL}$ insulin in a temperature-controlled flow chamber (flow rate $\sim 2 \mathrm{~mL} / \mathrm{min}$ ) equipped with platinum electrodes mounted on an Olympus IX-70 inverted microscope with a $40 \times$ objective. Cells were field-stimulated at $2 \mathrm{~Hz}$ frequency. Data were collected with an IonOptix FSI A/D board and IonWizard 6.2.2.61 software with SarcLen modules to determine sarcomere length. Fura-2 fluorescence was measured ratiometrically at $510 \mathrm{~nm}$ subsequent to alternate excitations at 340 and $380 \mathrm{~nm}$. Background fluorescence was subtracted for each excitation wavelength. The ratio of fluorescence intensities excited at $340 \mathrm{~nm}$ and $380 \mathrm{~nm}$ was used as a relative measurement of cytoplasmic $\mathrm{Ca}^{2+}$, and the ratio transient was fitted by the IonWizard monotonic transient analysis software.

In vivo muscle analysis. The function of the gastrocnemius muscle complex was studied in vivo on 57-62-day-old WT (6 male and 2 female) and $T_{t n}{ }^{\Delta C 1-2}$ (7 male and 2 female) mice using a Mouse Muscle Physiology System (model 809B; Aurora Scientific Inc.). Mice were anesthetized using isoflurane and placed on a heated platform $\left(39^{\circ} \mathrm{C}\right)$. Hair was removed from the right hind-leg and the knee immobilized using a noninvasive clamp. The foot was secured to the footplate on the force transducer with adhesive tape and set at a $90^{\circ}$ angle. Needle electrodes were placed just under the skin on either side of the tibial nerve, distal to the knee. Forces were recorded using ASI 610A Dynamic Muscle Control v5.5 software. The Isometric force-frequency relationship was measured by delivering optimal current at $1,10,20,30,40,60,80,100,125$, and $150 \mathrm{~Hz}$ stimulation frequencies. Maximal tetanic force was achieved at $150 \mathrm{~Hz}$. For obtaining the force-frequency relationship, both raw and normalized data were grouped based on genotype and results were fit with a with a four-parameter Hill equation. Tissue weights of the contralateral gastrocnemius complex (gastrocnemius, plantaris, and soleus) were used for force normalization.

Skinned myocytes mechanics. LV myocytes were isolated as explained above (see 'Cell Isolation and calcium transients') and were then skinned. Myocytes were skinned for $7 \mathrm{~min}$ in relaxing solution ((in mmol/L) $40 \mathrm{BES}, 10 \mathrm{EGTA}, 6.56 \mathrm{MgCl}_{2}$ $5.88 \mathrm{Na}$-ATP, 1.0 DTT, $46.35 \mathrm{~K}$-propionate, 15 creatine phosphate, $\mathrm{pH}$ 7.0) with protease inhibitors ((in mmol/L) 0.4 leupeptin, $0.1 \mathrm{E} 64$, and $0.5 \mathrm{PMSF}$ ) and $0.3 \%$ Triton X-100 (Ultrapure; Thermo Fisher Scientific). Cells were washed extensively with relaxing solution pCa 9 and stored on ice. Myocytes were added to a room temperature flow-through chamber mounted on the stage of an inverted microscope (Diaphot 200; Nikon). Skinned myocyte was glued at one end to a force transducer (Model 406A or 403A, Aurora Scientific). The other end was bent with a pulled glass pipette attached to micromanipulator so that the myocyte axis aligned with the microscope optical axis and cross-sectional area (CSA) was measured directly. The cross-sectional images of skinned cells were analyzed by ImageJ 1.41 software (National Institutes of Health) and were used to convert measured force to stress and for cell dimension study (Supplementary Fig. 4d). Then, the free end of the cell was glued to a servomotor (Model 308B, Aurora Scientific) that imposes controlled stretches. Sarcomere length (SL) was measured with a MyocamS and SarcLen acquisition module (IonWizard 6.2, IonOptix Co., MA, USA) attached to a computer. Passive tension was measured in relaxing solution pCa 9 with protease inhibitors at room temperature. Cells were stretched from their slack length at a speed of 1 base length/s followed by a $20 \mathrm{~s}$ hold and then a release back to the original length. The recovery time of at least $7 \mathrm{~min}$ in between stretches was utilized to prevent memory effects in subsequent measurements. Data were collected using a custom LabVIEW VI (National Instruments, Austin, TX, USA) at a sample rate of $1 \mathrm{kHz}$. Measured forces were converted to stress (force/unit undeformed CSA). The stress during the 1 base length/s stretch 
was plotted against the SL and fitted with a monoexponential curve to derive stress-SL relationships.

Skinned muscle fiber bundle mechanics. A total of 5 WT and $5 T t n^{\Delta C 1-2}$ mice (3 males and 2 females in each group) at 60 days of age were used in the skinned muscle mechanics experiments. Fiber bundles from skinned EDL tissue were dissected for mechanics experiments and mounted using aluminum $T$ clips between a length motor and a force transducer in an 802D Permeabilized Fiber Test Apparatus (Aurora Scientific Inc.) on a Zeiss Axio Observer A1 inverted microscope. SL was set using a high-speed VSL camera controlled by ASI 600A software (Aurora Scientific). Fiber bundles were maintained at $8{ }^{\circ} \mathrm{C}$, and the temperature was temporarily increased to $15^{\circ} \mathrm{C}$ during activation. Fibers were placed in relaxing solution (pCa 8.5), then preactivated in relaxing solution with reduced $1 \mathrm{mM}$ EGTA, and activated in pCa 4.5 activating solution ((in mM): $40 \mathrm{BES}, 10 \mathrm{CaCO}_{3}$-EGTA, $6.29 \mathrm{MgCl}_{2}, 6.12 \mathrm{Na}$-ATP, 1 DTT, 45.3 K-propionate, 15 creatine phosphate, and protease inhibitors) at $\mathrm{SL}=2.4$ $\mu \mathrm{m}$ to record maximal active tension. The resting slack SL was readjusted to $2.4 \mu \mathrm{m}$ after each activation-relaxation cycle. Specific force was expressed as force per CSA assuming elliptical fiber cross-section. Most activations had internal SL shortening during force development and in some fiber bundles an initial shortening phase was followed by a slow stretch phase (most likely due to sarcomeres outside the field of view that were stronger and that continued to shorten) that was accompanied by a slow force creep. In those cases force was recorded prior to the onset of the creep phase. To establish the force-SL relationship on the descending limb, fiber bundles were sequentially activated at a range of sarcomere lengths and SL was recorded both prior to and during activation. The passive force-SL curves were determined based on the average of pre- and post-activation passive forces for each SL. Internal shortening correction was applied to passive forces during activation by using nonlinear standard curve interpolation. The passive force during activation was then subtracted from the maximal active force, isolating the active force generating potential for each fiber bundle at a given SL.

Statistics. For animal studies the sample size was calculated by performing a power analysis using $\mathrm{G}^{\star}$ Power 3.1.9.2 software (written by Franz Faul, Universität Kiel, Germany). The genotype of the animals included in the study was determined by sequence-specific PCR (see Methods/Generation of the Ttn ${ }^{\Delta C 1-2}$ mouse model) as well as protein gel electrophoresis after the experiment (see Methods/Quantification of protein expression). Sample randomization as well as blinding of the investigator was applied in the case of in vivo animal studies, isolated cell experiments, and skinned muscle mechanics where investigators were unaware of the genotype of the animals during the experiments. Statistical analysis was performed using GraphPad Prism 6 or 7 (GraphPad Software, Inc.). Descriptive statistical results are shown as mean \pm s.e.m. unless stated otherwise. Differences between groups were considered to be statistically significant at a probability value of $p<0.05$. A two-tailed $t$-test was used when comparing two groups only, and Welch's correction was applied in the case of unequal variances between the two groups. One-way analysis of variance using Bonferroni post hoc analysis was performed to assess differences between multiple groups. In order to increase the statistical power of the tests equal or close to equal sample size was applied within independent groups. Linear regression analysis was used to fit and compare EM, IEM, and SR-SIM epitope distance data (Fig. 2). Nonlinear curve fit was applied to the growth curves of animals (Supplementary Fig. 1C) and to the summarized shrinkage data of cardiac (Supplementary Fig. 4B) and skeletal (Supplementary Fig. 5B) muscle. Functional tests required both linear and nonlinear regression analyses to be performed (Fig. 3a-d). The genotype ratio of offspring from $\mathrm{Het} \times \mathrm{Het}$ breeding was studied by $\chi^{2}$ test (Supplementary Fig. 1B). For detailed statistical information see Supplementary Table 4 .

Data availability. All data generated or analyzed during this study are included in this published article (and its supplementary information files) and are available from the corresponding author on reasonable request.

Received: 16 March 2017 Accepted: 22 August 2017

Published online: 19 October 2017

\section{References}

1. Zoghbi, M. E., Woodhead, J. L., Moss, R. L. \& Craig, R. Three-dimensional structure of vertebrate cardiac muscle myosin filaments. Proc. Natl Acad. Sci. USA 105, 2386-2390 (2008).

2. Squire, J. M., Paul, D. M. \& Morris, E. P. Myosin and actin filaments in muscle: structures and interactions. Subcell. Biochem. 82, 319-371 (2017).

3. Sosa, H., Popp, D., Ouyang, G. \& Huxley, H. E. Ultrastructure of skeletal muscle fibers studied by a plunge quick freezing method: myofilament lengths. Biophys. J. 67, 283-292 (1994).
4. Gordon, A. M., Huxley, A. F. \& Julian, F. J. The variation in isometric tension with sarcomere length in vertebrate muscle fibres. J. Physiol. 184, 170-192 (1966).

5. Whiting, A., Wardale, J. \& Trinick, J. Does titin regulate the length of muscle thick filaments? J. Mol. Biol. 205, 263-268 (1989).

6. Wang, K. Titin/connectin and nebulin: giant protein rulers of muscle structure and function. Adv. Biophys. 33, 123-134 (1996).

7. Tskhovrebova, L. \& Trinick, J. Titin and nebulin in thick and thin filament length regulation. Subcell. Biochem. 82, 285-318 (2017).

8. Bennett, P. M. \& Gautel, M. Titin domain patterns correlate with the axial disposition of myosin at the end of the thick filament. J. Mol. Biol. 259, 896-903 (1996).

9. Furst, D. O., Osborn, M., Nave, R. \& Weber, K. The organization of titin filaments in the half-sarcomere revealed by monoclonal antibodies in immunoelectron microscopy: a map of ten nonrepetitive epitopes starting at the $\mathrm{Z}$ line extends close to the M line. J. Cell Biol. 106, 1563-1572 (1988).

10. Labeit, S. \& Kolmerer, B. Titins: giant proteins in charge of muscle ultrastructure and elasticity. Science 270, 293-296 (1995).

11. Granzier, H. L. \& Labeit, S. Titin and its associated proteins: the third myofilament system of the sarcomere. Adv. Protein Chem. 71, 89-119 (2005).

12. Hidalgo, C. \& Granzier, H. Tuning the molecular giant titin through phosphorylation: role in health and disease. Trends Cardiovasc. Med. 23, 165-171 (2013).

13. Linke, W. A. \& Hamdani, N. Gigantic business: titin properties and function through thick and thin. Circ. Res. 114, 1052-1068 (2014).

14. Freiburg, A. et al. Series of exon-skipping events in the elastic spring region of titin as the structural basis for myofibrillar elastic diversity. Circ. Res. 86, 1114-1121 (2000).

15. Herman, D. S. et al. Truncations of titin causing dilated cardiomyopathy. $N$. Engl. J. Med. 366, 619-628 (2012).

16. Schafer, S. et al. Titin-truncating variants affect heart function in disease cohorts and the general population. Nat. Genet. 49, 46-53 (2017).

17. Ware, J. S. et al. Shared genetic predisposition in peripartum and dilated cardiomyopathies. N. Engl. J. Med. 374, 233-241 (2016).

18. Franaszczyk, M. et al. Titin truncating variants in dilated cardiomyopathy prevalence and genotype-phenotype correlations. PLoS ONE 12, e0169007 (2017).

19. Granzier, H. L. et al. Deleting titin's I-band/A-band junction reveals critical roles for titin in biomechanical sensing and cardiac function. Proc. Natl Acad. Sci. USA 111, 14589-14594 (2014).

20. Furst, D. O., Nave, R., Osborn, M. \& Weber, K. Repetitive titin epitopes with a $42 \mathrm{~nm}$ spacing coincide in relative position with known A band striations also identified by major myosin-associated proteins. An immunoelectronmicroscopical study on myofibrils. J. Cell Sci. 94, 119-125 (1989).

21. Muhle-Goll, C. et al. Structural and functional studies of titin's fn 3 modules reveal conserved surface patterns and binding to myosin S1--a possible role in the Frank-Starling mechanism of the heart. J. Mol. Biol. 313, 431-447 (2001)

22. Freiburg, A. \& Gautel, M. A molecular map of the interactions between titin and myosin-binding protein C. Implications for sarcomeric assembly in familial hypertrophic cardiomyopathy. Eur. J. Biochem. 235, 317-323 (1996).

23. Tskhovrebova, L., Bennett, P., Gautel, M. \& Trinick, J. Titin ruler hypothesis not refuted. Proc. Natl Acad. Sci. USA 112, E1172 (2015).

24. Granzier, H. L. Reply to Tskhovrebova et al.: titin's IA junction does not control thick filament length. Proc. Natl Acad. Sci. USA 112, E1173 (2015).

25. Yancy, C. W. et al. 2013 ACCF/AHA guideline for the management of heart failure: a report of the american college of cardiology foundation/american heart association task force on practice guidelines. J. Am. Coll. Cardiol. 62, e147-e239 (2013).

26. Hershberger, R. E., Hedges, D. J. \& Morales, A. Dilated cardiomyopathy: the complexity of a diverse genetic architecture. Nat. Rev. Cardiol. 10, 531-547 (2013).

27. Roberts, A. M. et al. Integrated allelic, transcriptional, and phenomic dissection of the cardiac effects of titin truncations in health and disease. Sci. Transl. Med 7, 270 ra276 (2015).

28. Norton, N. et al. Exome sequencing and genome-wide linkage analysis in 17 families illustrate the complex contribution of TTN truncating variants to dilated cardiomyopathy. Circ. Cardiovasc. Genet. 6, 144-153 (2013).

29. Lee, K., Harris, S. P., Sadayappan, S. \& Craig, R. Orientation of myosin binding protein $\mathrm{C}$ in the cardiac muscle sarcomere determined by domain-specific immuno-EM. J. Mol. Biol. 427, 274-286 (2015).

30. Pollack, G. H. The cross-bridge theory. Physiol. Rev. 63, 1049-1113 (1983).

31. Burkhoff, D., Mirsky, I. \& Suga, H. Assessment of systolic and diastolic ventricular properties via pressure-volume analysis: a guide for clinical, translational, and basic researchers. Am. J. Physiol. Heart Circ. Physiol. 289, H501-H512 (2005)

32. Japp, A. G., Gulati, A., Cook, S. A., Cowie, M. R. \& Prasad, S. K. The diagnosis and evaluation of dilated cardiomyopathy. J. Am. Coll. Cardiol. 67, 2996-3010 (2016) 
33. LeWinter, M. M., Wu, Y., Labeit, S. \& Granzier, H. Cardiac titin: structure, functions and role in disease. Clin. Chim. Acta 375, 1-9 (2007).

34. Granzier, H., Wu, Y., Siegfried, L. \& LeWinter, M. Titin: physiological function and role in cardiomyopathy and failure. Heart Fail. Rev. 10, 211-223 (2005).

35. Cazorla, O. et al. Differential expression of cardiac titin isoforms and modulation of cellular stiffness. Circ. Res. 86, 59-67 (2000).

36. Neagoe, C., Opitz, C. A., Makarenko, I. \& Linke, W. A. Gigantic variety: expression patterns of titin isoforms in striated muscles and consequences for myofibrillar passive stiffness. J. Muscle Res. Cell Motil. 24, 175-189 (2003).

37. Bennett, P., Craig, R., Starr, R. \& Offer, G. The ultrastructural location of Cprotein, X-protein and H-protein in rabbit muscle. J. Muscle Res. Cell Motil. 7, 550-567 (1986).

38. Granzier, H. L. \& Irving, T. C. Passive tension in cardiac muscle: contribution of collagen, titin, microtubules, and intermediate filaments. Biophys. J. 68, 1027-1044 (1995)

39. Chung, C. S. et al. Shortening of the elastic tandem immunoglobulin segment of titin leads to diastolic dysfunction. Circulation 128, 19-28 (2013).

40. Radke, M. H. et al. Targeted deletion of titin N2B region leads to diastolic dysfunction and cardiac atrophy. Proc. Natl Acad. Sci. USA 104, 3444-3449 (2007).

41. Methawasin, M. et al. Experimentally increasing titin compliance in a novel mouse model attenuates the Frank-Starling mechanism but has a beneficial effect on diastole. Circulation 129, 1924-1936 (2014).

42. Hinze, F., Dieterich, C., Radke, M. H., Granzier, H. \& Gotthardt, M. Reducing RBM20 activity improves diastolic dysfunction and cardiac atrophy. J. Mol. Med. 94, 1349-1358 (2016)

43. Davis, J. et al. A tension-based model distinguishes hypertrophic versus dilated cardiomyopathy. Cell 165, 1147-1159 (2016).

44. Sheikh, F. et al. An FHL1-containing complex within the cardiomyocyte sarcomere mediates hypertrophic biomechanical stress responses in mice. J. Clin. Invest. 118, 3870-3880 (2008).

45. Begay, R. L. et al. Role of titin missense variants in dilated cardiomyopathy. J. Am. Heart Assoc. 4, e002645 (2015).

46. Dobin, A. et al. STAR: ultrafast universal RNA-seq aligner. Bioinformatics 29, 15-21 (2013).

47. Hidalgo, C. et al. PKC phosphorylation of titin's PEVK element: a novel and conserved pathway for modulating myocardial stiffness. Circ. Res. 105, 631-638 (2009). $617 \mathrm{p}$ following 638.

48. Warren, C. M., Jordan, M. C., Roos, K. P., Krzesinski, P. R. \& Greaser, M. L. Titin isoform expression in normal and hypertensive myocardium. Cardiovasc. Res. 59, 86-94 (2003).

49. Futaesaku, Y. \& Mizuhira, V. New fixation for biological membranes using tannic acids. Acta Histochem. Cytochem. 5, 233-236 (1972).

50. Lawn, A. M. The use of potassium permanganate as an electron-dense stain for sections of tissue embedded in epoxy resin. J. Biophys. Biochem. Cytol. 7, 197-198 (1960)

51. Granzier, H., Kellermayer, M., Helmes, M. \& Trombitas, K. Titin elasticity and mechanism of passive force development in rat cardiac myocytes probed by thin-filament extraction. Biophys. J. 73, 2043-2053 (1997).

52. Gregorio, C. C. et al. The NH2 terminus of titin spans the Z-disc: its interaction with a novel $19-\mathrm{kD}$ ligand (T-cap) is required for sarcomeric integrity. J. Cell Biol. 143, 1013-1027 (1998).
53. Trombitas, K. et al. Molecular basis of passive stress relaxation in human soleus fibers: assessment of the role of immunoglobulin-like domain unfolding. Biophys. J. 85, 3142-3153 (2003).

54. Methawasin, M. et al. Experimentally increasing titin compliance in a novel mouse model attenuates the Frank-Starling mechanism but has a beneficial effect on diastole. Circulation 129, 1924-1936 (2014).

\section{Acknowledgements}

Dr. Granzier is the Allan and Alfie Norville Endowed Chair. We are grateful to our lab members Xiangdang Liu, Xiaoqun Zhou, Chandra Saripalli, Maya Adler, Yaeren Hernandez, and Alexander Gasch, the University of Arizona Genetic Engineering of Mouse Model and the Mouse Phenotyping Core Facilities. We are grateful to Dr Carol Gregorio for the I103 antibody and Dr Samantha Harris for the cMyBP-C (C5-C7) antibody. Funding was by Foundation Leducq (TNE-13CVD04) and National Institutes of Health Grants HL062881, HL118524, and HL115988. We dedicate this study to Dr Károly (Charles) Trombitás, a pioneer in electron microscopy on titin and a continued source of inspiration.

\section{Author contributions}

P.T., B.K., J.S., M.M., J.E.S.III, J.K., and H.G.: experimental design, data collection, analysis, and writing of manuscript. S.L.:experimental design, reagents, and writing of manuscript.

\section{Additional information}

Supplementary Information accompanies this paper at doi:10.1038/s41467-017-01144-9.

Competing interests: The authors declare no competing financial interests.

Reprints and permission information is available online at http://npg.nature.com/ reprintsandpermissions/

Publisher's note: Springer Nature remains neutral with regard to jurisdictional claims in published maps and institutional affiliations.

(c) (i) Open Access This article is licensed under a Creative Commons Attribution 4.0 International License, which permits use, sharing, adaptation, distribution and reproduction in any medium or format, as long as you give appropriate credit to the original author(s) and the source, provide a link to the Creative Commons license, and indicate if changes were made. The images or other third party material in this article are included in the article's Creative Commons license, unless indicated otherwise in a credit line to the material. If material is not included in the article's Creative Commons license and your intended use is not permitted by statutory regulation or exceeds the permitted use, you will need to obtain permission directly from the copyright holder. To view a copy of this license, visit http://creativecommons.org/ licenses/by/4.0/.

(C) The Author(s) 2017 